\title{
UPPER CUT-OFF FREQUENCY OF THE BOUND WAVE AND NEW LEAKY WAVE ON THE SLOTLINE
}

\author{
Ján Zehentner, Jan Macháč, Maurizio Migliozzi \\ Czech Technical University, Technická 2, 16627 Prague 6, Czech Republic
}

\begin{abstract}
This paper contributes to a more profound understanding of uniplanar circuit behaviour. A simple closed-form formula providing the upper cut-off frequency of the bound wave propagating over the slotline is presented. A new leaky wave which brings down this limit due to the overlapping of the boundand leaky-mode regions is identified and reported. This wave can deteriorate circuit performance, especially in the mm-wave band. Conclusions made for the slotline hold generally for open printed-circuit lines.
\end{abstract}

\section{INTRODUCTION}

Planar transmission lines used in microwave, millimetre wave and optical integrated circuits have been investigated intensively over the last fifteen years, e.g. [1-5]. Besides the attempt to minimize natural losses in them, attention has been paid to leakage that can result in cross-talk between neighbouring components or portions of the circuit, in a lowering of their quality factors, or in outright loss of power. Leakage has a significant effect on circuit performance. Shigesawa, Tsuji and Oliner [4] have summarized all earlier findings about effects which may occur in open printed-circuit lines.

In this paper we will deal with a new, previously unidentified leaky wave on the slotline, with its influence on the upper limit of the dominant bound wave frequency band, and will present closed-form formulae for this limit suitable for CAD. The paper is a contribution to the general discussion on characteristics of open planar transmission lines, particularly the slotline.

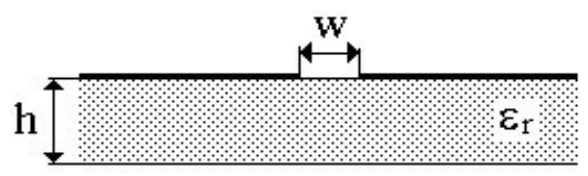

Fig. 1 Cross-section of the slotline

We have analysed the open slotline, Fig. 1 , by the method of moments modified as in the Galerkin testing procedure in the spectral domain with successive complex root searching. The integration path in the spectral domain was deformed from the real axis to include the residue contributions associated with the propagation of the corresponding surface waves. The accuracy of our calculation was checked by comparison of the normalized phase constant $\beta / k_{0}$ and the normalized leakage constant $\alpha / k_{0}$ with their plotting published in [4]. Thus, the program provides us with all solutions known and published until now.

\section{THE 2ND LEAKY WAVE}

However, besides these solutions the dispersion equation of the slotline also has a further solution that is new and interesting. Let us denote its already known complex solution as 
the 1st leaky mode, and the new complex solution, which we are reporting here, as the 2 nd leaky mode. The behaviour of the latter is illustrated in Fig. 2, and belongs to the slotline taken over from [4]. Between the cut-off frequency of the $T E_{1}$ surface wave $f_{1}$ and $f_{2}$ when the 2nd leaky mode sets in, the solution is improper real. Between $f_{2}$ and $f_{3}$, at which point the phase constant of the 2nd leaky wave becomes equal to that of the $\mathrm{TE}_{1}$ surface wave, the leaky wave is nonphysical in the sense of the generalized condition of leakage [5]. By chance $f_{3}(0.2695)$ is here only slightly lower than $f_{4}$ $(0.2780)$, the uper cut-off frequency of the pure dominant bound wave. The conventional frequency gap from $f_{4}$ to $f_{5}$ now disappears due to the presence of the 2nd leaky wave. At frequencies higher than $f_{3}$ energy can leak into the $\mathrm{TE}_{1}$ and $\mathrm{TM}_{0}$ surface waves. Leakage to the $\mathrm{TE}_{1}$ surface wave occurs at a lower angle than to the $\mathrm{TM}_{0}$ surface wave. Corresponding leakage constant $a_{2}$ is greater than $a_{1}$, Figs. 2,3,4.

When w/h increases, e.g. to $0.5, f_{3}$ is distinctly lower than $f_{4}$ and the bound and the 2nd leaky wave are present simultaneously in this overlapping region, Fig. 3 . From $\mathrm{f}_{6}$ to $\mathrm{f}_{7}$ the wave is nonphysical since $b / k_{0}$ does not comply with the generalized condition of leakage [5].

These newly-revealed characteristics are more expressive on the higher permittivity substrate, e.g. when $\mathrm{e}_{\mathrm{r}}=10.8, \mathrm{w} / \mathrm{h}=0.236(\mathrm{w}=0.15$ $\mathrm{mm}$ and $\mathrm{h}=0.635 \mathrm{~mm})$, Fig. 4. Now the overlapping interval from $\mathrm{f}_{3}=46.0 \mathrm{GHz}$ to $\mathrm{f}_{4}=59.9 \mathrm{GHz}$ is sufficiently significant for practical purposes. The 2nd solution either improper real Figs. 2,3, or improper complex Fig. 5, always sets in at the $\mathrm{TE}_{1}$ cut-off frequency $f_{1}$ and has the same value as the 1 st leaky wave at this split-off point. The 2nd leaky wave has much greater influence on the behaviour of the slotline of a narrower slotwidth Figs. 2,3,4, while for a wider slotwidth its influence is negligible, since $f_{3}$ and $f_{6}$ are too close together, Fig. 5.

\section{UPPER CUT-OFF FREQUENCY OF THE BOUND WAVE}

To avoid trouble caused by surface leakage it is necessary to know the upper frequency cut-off of the dominant bound wave. In the case of a spectral gap this is given by $f_{4}$ denoted hereafter as $f_{c}$. When simultaneous propagation of the bound and the 1st leaky wave occurs, the upper frequency cut-off $\mathrm{f}_{\mathrm{cl}}$ is equal to $f_{5}$ as in Fig. 5. If the region of the bound and the 2nd leaky wave overlap, the cut-off frequency $f_{c 2}$ is given by $f_{3}$, e.g. Fig. 4.

The normalized upper frequency cut-off $\left(\mathrm{h} / \lambda_{\mathrm{c}}\right)$ instead of $\mathrm{f}_{\mathrm{c}}$ depending on the relative dimension $w / h$ and permittivity $\varepsilon_{r}$ is shown in Fig. 6. Designers will appreciate the closed-form formula

$$
\left(\mathrm{h} / \lambda_{\mathrm{c}}\right)=\mathrm{A}_{1}\left[\mathrm{~A}_{2}+\mathrm{A}_{3}(\mathrm{w} / \mathrm{h})^{\mathrm{A}_{4}}\right]^{\mathrm{A}_{5}}
$$

where $A_{1}, A_{2}, A_{3}, A_{4}, A_{5}$ are simple functions of $\varepsilon_{r}$. Owing to limited space in this paper, the complete records will be given in the talk. Eqn. 1 holds when $0.01 \leq \mathrm{w} / \mathrm{h} \leq 1$ and $2 \leq \varepsilon_{\mathrm{r}} \leq 16$. These intervals of validity are the same also for Eqns. 3 and 5.

The equation

$$
\begin{aligned}
\left(\mathrm{h} / \lambda_{\mathrm{cb} 1}\right) & =[0.76388+0.20999 \\
& \left.\cdot(\mathrm{w} / \mathrm{h}-0.493)^{0.08212}\right]^{24.589}
\end{aligned}
$$

satisfies the condition $\mathrm{f}_{\mathrm{c}}=\mathrm{f}_{\mathrm{cl}}$ and controls the boundary line separating the left pure bound wave region from the right region of simultaneous propagation of the bound and the 1 st leaky waves.

Similarly, normalized $\left(\mathrm{h} / \lambda_{\mathrm{c} 1}\right)$ instead of $\mathrm{f}_{\mathrm{c} 1}$ is also plotted in Fig. 6. The formula 
$\left(h / \lambda_{c 1}\right)=\left[B_{1}+B_{2}(w / h)^{-B_{3}}\right]^{2}$

describes $f_{c l} . B_{1}, B_{2}, B_{3}$, the $\varepsilon_{r}$ dependent functions will be specified in the talk.

The condition $f_{c}=f_{c 2}$ produces the equation

$$
\begin{aligned}
\left(\mathrm{h} / \lambda_{\mathrm{cb} 2}\right) & =[0.00816+0.14095 \\
& \left.\cdot(\mathrm{w} / \mathrm{h}-0.037)^{0.68218}\right]^{0.49415}
\end{aligned}
$$

defining the boundary line separating the left pure bound wave region from the right region of simultaneous propagation of the bound wave and the 2 nd leaky wave, Fig. 7.

The normalized upper frequency cut-off $\left(\mathrm{h} / \lambda_{\mathrm{c} 2}\right)$ instead of $\mathrm{f}_{\mathrm{c} 2}$ is also drawn in Fig. 7 and

$$
\left(h / \lambda_{c 2}\right)=\left[C_{1}+C_{2}(w / h)^{-C_{3}}\right]^{C_{4}}+C_{5}
$$

where $\mathrm{C}_{1}, \mathrm{C}_{2}, \mathrm{C}_{3}, \mathrm{C}_{4}, \mathrm{C}_{5}$ are $\varepsilon_{\mathrm{r}}$ dependent functions. Their reading will be given in the talk. It stands to reason that either the 1 st or the 2 nd leaky wave brings down the upper frequency limit of the bound wave.

\section{CONCLUSIONS}

We believe that the new effects revealed on the slotline may also occur on other open printed-circuit lines and possess generality encoded in the multi-valued solution of their dispersion equations. This paper presents new findings regarding the behaviour of the slotline in the mm-wave band. It also provides useful formulae for CAD, particularly in determining the desired operation frequency band in which only the bound wave can be propagated.

\section{ACKNOWLEDGMENTS}

We gratefully acknowledge the scholarship granted by the Faculty of Electrical Engineering of the Czech Technical University in Prague to M. Migliozzi, a student of the University of Ancona, Italy, supporting his participation in this research. The work was done on the SP-2 computer at the Joint Supercomputer Center of the Czech Technical University, University of Chemical Technology and IBM Prague.

\section{REFERENCES}

[1] A. A. Oliner: Leakage from higher modes on microstrip line with application to antennas. Radio Science, Vol. 22, 1987, Number 6, pp. 907-912.

[2] H. Shigesawa, M. Tsuji, A. A. Oliner: Dominant mode power leakage from printed-circuit waveguides. Radio Science, Vol. 26, 1991, Number 2, pp. 559-564.

[3] A. A. Oliner, D. R. Jackson: On spectral gaps at the transition between bound and leaky modes. Proceedings of the 1995 International Symposium on Electromagnetic Theory, URSI, St. Petersburg, Russia, May 23-26, 1995, pp. 764-766.

[4] H. Shigesawa, M. Tsuji, A. A. Oliner: Simultaneous Propagation of Bound and Leaky Dominant Modes on Printed-Circuit Lines: A New General Effect. IEEE Trans. Microwave Theory Tech., Vol. 43, December 1995, pp. 3007-3019.

[5] D. Nghiem, J. T. Williams, D. R. Jackson, K. A. Michalski: The Choice of Integration Path in the SpectralDomain Solution of Leaky Modes. Personal communication about the viewgraphs presented at the URSI National Radio Science Meeting, Boulder, Colorado, January 1995 by D. R. Jackson.
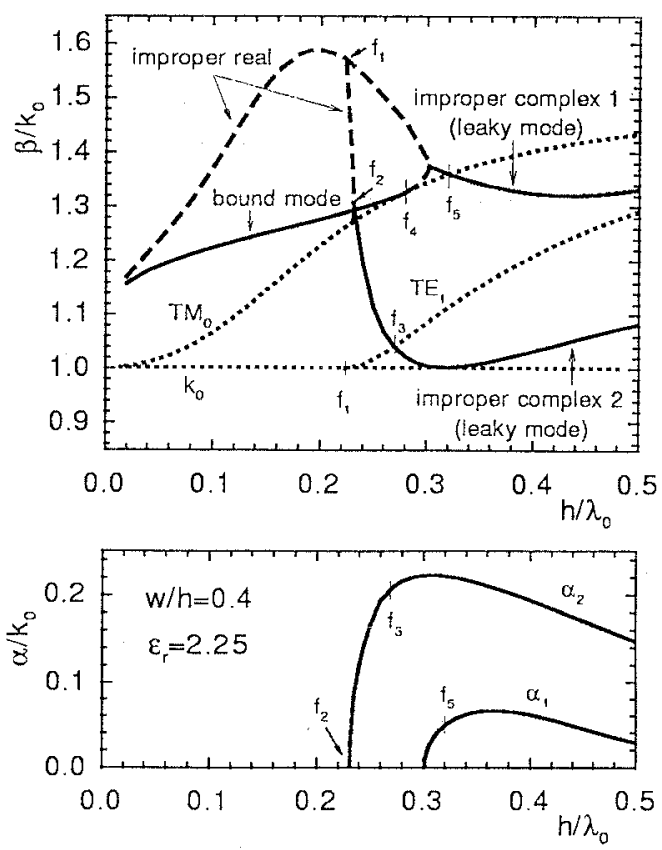

Fig. 2 The normalized phase and leakage constants for the slotline with $\mathrm{w} / \mathrm{h}=0.4$ and $\varepsilon_{\mathrm{r}}=2.25$ as a function of normalized frequency $\left(h / \lambda_{0}\right)$. 

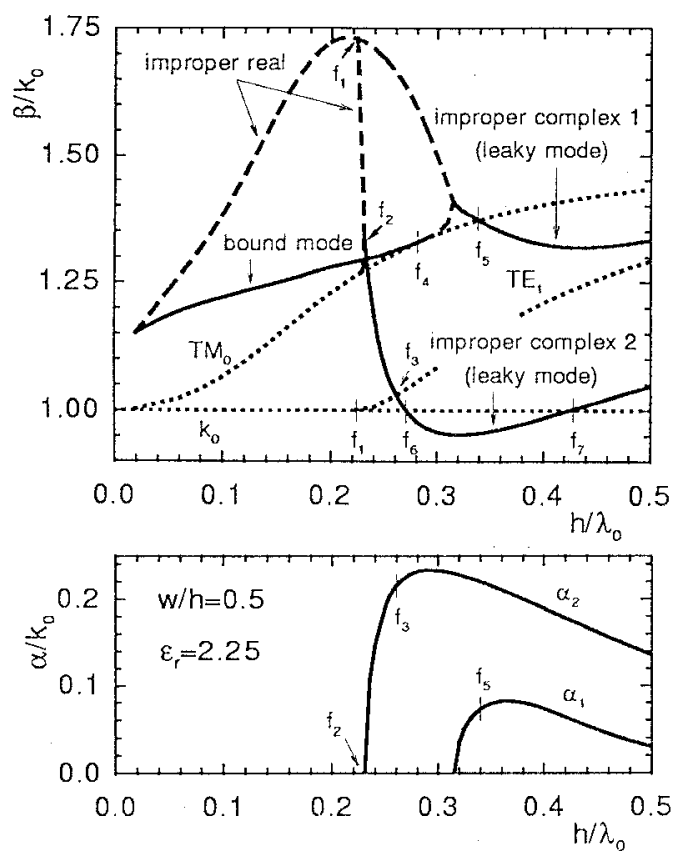

Fig. 3 A plot similar to that in Fig. 2 but for wider slotwidth $\mathrm{w} / \mathrm{h}=0.5$.
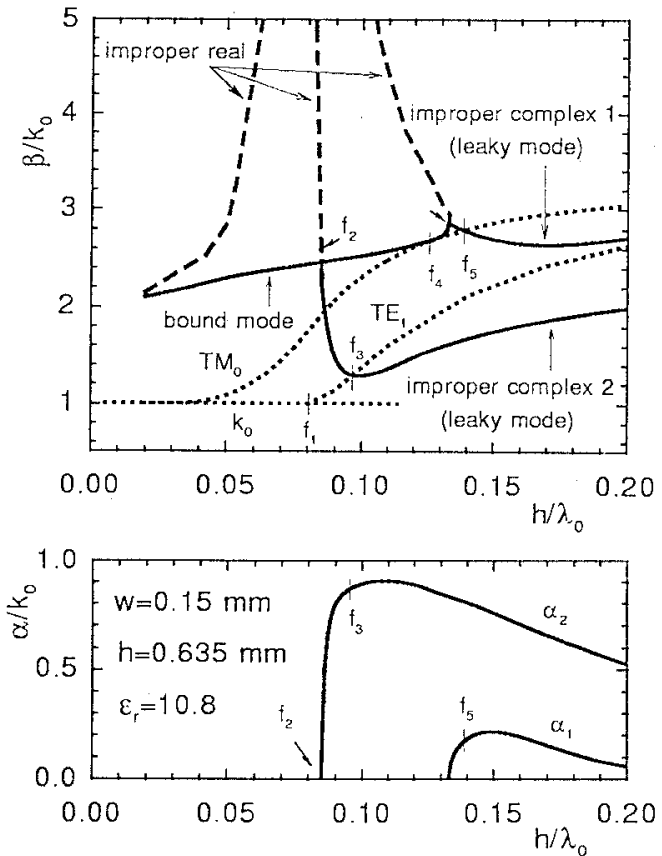

Fig. $4 \mathrm{~A}$ plot similar to that in Fig. 2 but for narrower slotwidth $\mathrm{w} / \mathrm{h}=0.236$ and higher substrate permittivity $\varepsilon_{\mathrm{r}}=10.8$.

Fig. 7 A plot similar to that in Fig. 6 but the normalized upper frequency cut-off of the dominant bound wave $\left(h / \lambda_{c 2}\right)$ is now determined by simultaneous propagation of the 2 nd leaky wave .
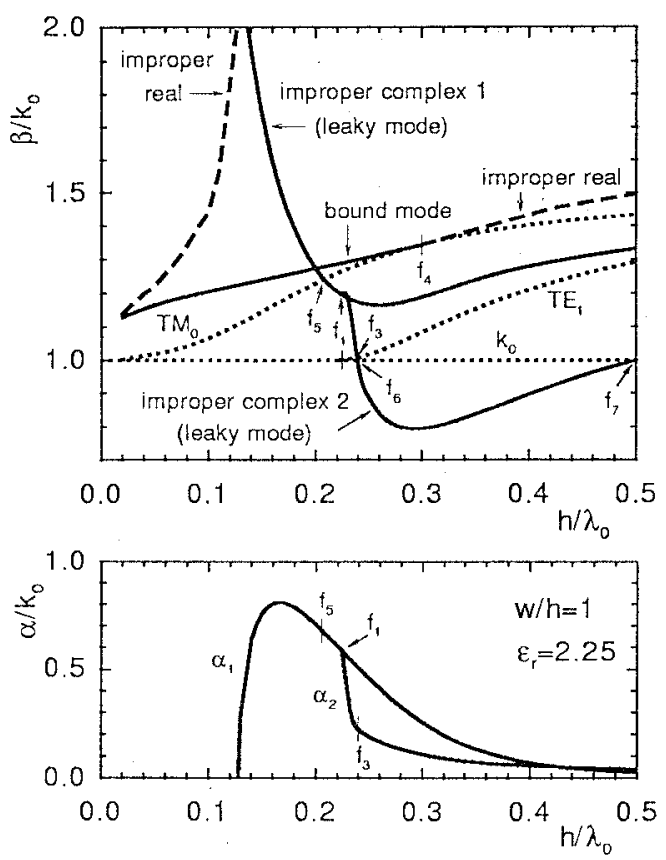

Fig. 5 A plot similar to that in Fig. 3 but for still wider slotwidth $\mathrm{w} / \mathrm{h}=1$.

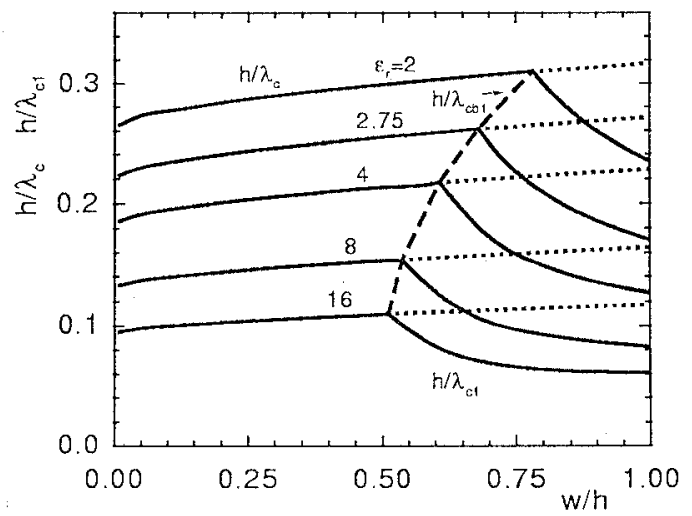

Fig. 6 The normalized upper frequency cut-off of the dominant bound wave determined by the spectral gap $\left(\mathrm{h} / \lambda_{0}\right)$ and by simultaneous propagation of the lst leaky wave $\left(h / \lambda_{c 1}\right)$.

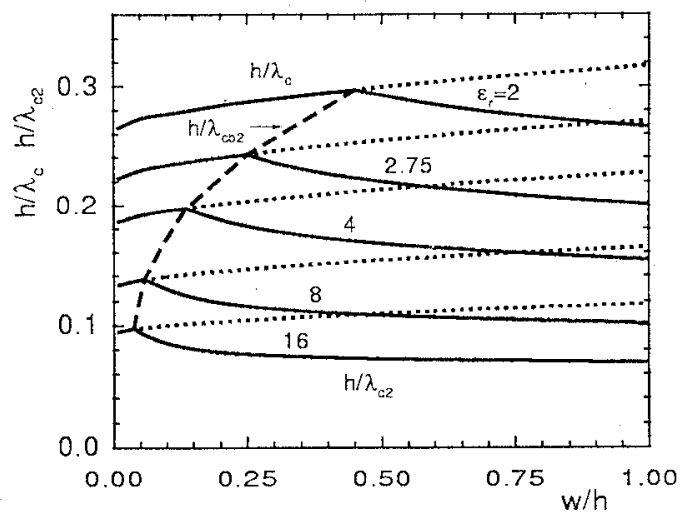

